\title{
Salidroside slows the progression of EA.hy926 cell senescence by regulating the cell cycle in an atherosclerosis model
}

\author{
LIN SUN $^{1 *}$, FANGFANG DOU $^{1 *}$, JIULIN CHEN $^{1}$, HUIYING CHI $^{1}$, \\ SANLI XING ${ }^{1}$, TE LIU ${ }^{1}$, SHENWEI SUN ${ }^{2}$ and CHUAN CHEN ${ }^{1}$ \\ ${ }^{1}$ Basic Research Department, Shanghai Geriatric Institute of Chinese Medicine and ${ }^{2}$ Internal Medicine of Traditional \\ Chinese Medicine, Longhua Hospital, Shanghai University of Traditional Chinese Medicine, Shanghai 200031, P.R. China
}

Received June 1, 2017; Accepted October 2, 2017

DOI: $10.3892 / \mathrm{mmr} .2017 .7872$

\begin{abstract}
Aging is the major risk factor for diseases of the cardiovascular system, such as coronary atherosclerotic heart disease, but little is known about the relationship between atherosclerosis (AS) and age-related declines in vascular structure and function. Here, we used histological analyses in combination with molecular biology techniques to show that lipid deposition in endothelial cell was accompanied by aging and growth arrest. Endothelial cell senescence is sufficient to cause AS; however, we found that salidroside reduced intracellular lipid deposition, slowed the progression of endothelial cell senescence and inhibited the expression of the senescence-related molecules and phosphorylated the retinoblastoma $(\mathrm{Rb})$ protein. Further study confirmed that salidroside increased the percent of $\mathrm{S}$ phase cells in oxidized low-density lipoprotein (ox-LDL)-treated endothelial cells. Collectively, vascular endothelial cell function declined with age and AS, and our data suggested that salidroside prevented ox-LDL-treated endothelial cell senescence by promoting cell cycle progression from G0/G1 phase to $\mathrm{S}$ phase via Rb phosphorylation. We demonstrated for the first time the complex interactions between AS and endothelial cell senescence, and we believe that salidroside represents a promising therapy for senescence-related AS.
\end{abstract}

Correspondence to: Professor Chuan Chen, Basic Research Department, Shanghai Geriatric Institute of Chinese Medicine, Shanghai University of Traditional Chinese Medicine, Building C, 365 South Xiangyang Road, Xuhui, Shanghai 200031, P.R. China E-mail: ch9453@126.com

Dr Shenwei Sun, Internal Medicine of Traditional Chinese Medicine, Longhua Hospital, Shanghai University of Traditional Chinese Medicine, 725 South Wanping Road, Xuhui, Shanghai 200031, P.R. China

E-mail: sunsw55@hotmail.com

*Contributed equally

Key words: salidroside, aging, atherosclerosis, endothelial cell, lipid deposition

\section{Introduction}

The effect of aging on atherosclerosis (AS) can be explained by the observation that the prevalence of AS increases progressively with age. Functional and morphological changes in healthy aged vascular tissue lead to decreases in arterial elasticity that affect reservoir function and pulse pressure in older adults and are considered a predictor of several cardiovascular outcomes, such as stroke and myocardial infarction (1). Age-related oxidative damage and vascular inflammation are the main causes of arterial dysfunction. Nitric oxide (NO) is the most important regulator of vascular endothelial function, and its bioavailability enables it to play a prominent role during the initial stages of AS, which are characterized by the combination of endothelial damage and lipid infiltration (2). Reactive oxygen species (ROS) induced activation of the transcription factor nuclear factor- $\kappa \mathrm{B}(\mathrm{NF}-\kappa \mathrm{B})$ promotes $\mathrm{AS}$ via the release of several cytokines and adhesion molecules, which collectively contribute to AS progression $(3,4)$. Recently, increasing amounts of evidence have linked vascular endothelial cell senescence to AS development $(5,6)$, but the biological mechanisms underlying this relationship need to be studied further. Migliaccio et al found that genetic $\mathrm{p} 66^{\text {Shc }}$ deletions decrease ROS levels and prolong mouse lifespans by $30 \%$ and that p53 and p21 mediated stress responses are impaired in p66 $6^{\text {shc-l- }}$ cells (7). p66 $6^{\text {Shc }}$ is also involved in the pathology of AS and is a key determinant of aging. p66 $6^{\text {Shc }}$ silencing attenuates oxidized low-density lipoprotein (ox-LDL)-induced ROS production in endothelial cells (8) and reduces plaque formation in $\mathrm{ApoE}^{-/}$mice subjected to a high-cholesterol diet (9). Here, we showed that ox-LDL-induced varieties in senescence-related protein occurred in conjunction with senescence-related increases in lipid deposition in EA.hy926 endothelial cell, but the biological mechanisms underlying the promotion of cell senescence have not been completely elucidated. Some studies have demonstrated that p16 levels gradually increase in senescent human diploid fibroblasts, reaching levels that are approximately 40 -fold higher than those of early passaged cells (10), and that increased cyclin-dependent kinase inhibitor 2A, isoform INK4a (p16INK4a) expression leads to retinal ganglion cell senescence in adult human retinas (11). It is known that p16 is the major cyclin-dependent kinase (CDK) inhibitor and that p16 upregulation is a key event in 
growth arrest-related cell senescence. It is now considered that p21 was involved in regulation of cell proliferation, differentiation, migration, senescence, and apoptosis (12). And recent report confirmed that p21-mediated cellular senescence was p53-independent (13). In ox-LDL-induced senescent EA.hy926 cells, the levels of p53, p21 and p16 mRNA and cellular protein expression were increased dramatically, the phosphorylated retinoblastoma $(\mathrm{Rb})$ level was decreased, and the cell cycle was arrested in G0/G1 phase. We confirmed that decreases in phosphorylated $\mathrm{Rb}$ protein levels may underlie the development of EA.hy926 cell senescence.

Salidroside, one of the active ingredients of Rhodiolacrenulata, can be used as an effective anti-diabetes agent (14), can protect against $A \beta$-induced neurotoxicity in transgenic Drosophila Alzheimer's disease (AD) models (15), and can also alleviate oxidative stress (16) and inflammation (17) in different cell and animal models; however, there are little data suggesting that salidroside protects against age-related AS. Xing et al showed that salidroside plays a critical role in promoting NO production and activating AMPK signaling pathway to improve endothelial function in AS (18) and Some study indicated that salidroside could attenuate $\mathrm{H}_{2} \mathrm{O}_{2}$ induced cell death by inhibiting caspase-3, 9 and cleavage of poly (ADP-ribose) polymerase (PARP) activation (19). Our previous study found that aging cells exist in atherosclerotic plaque of high-fat diet $\mathrm{ApoE}^{-/-}$mice, and salidroside could decrease the aging cells. In the present study, we investigated the protective effects of salidroside against ox-LDL-induced EA.hy926 cell senescence and explored the mechanisms underlying ox-LDL induced endothelial cell senescence. We showed that salidroside significantly protected cultured EA.hy926 cells against ox-LDL-induced cytotoxicity. Furthermore, we provided evidence that these effects were induced by changes in the expression of senescence-related molecular targets (p66, p53 and $\mathrm{p} 21$ ) in the phosphorylated $\mathrm{Rb}$ protein signaling pathway.

\section{Materials and methods}

EA.hy926 cells culture and proliferation assay. Human umbilical vein EA.hy926 cells (Cell bank, Shanghai Institute for Biological Science, Shanghai, China) were cultured in Dulbecco's modified Eagle medium (DMEM; Invitrogen, CA, USA) supplemented with $10 \%$ heat-inactivated fetal bovine serum (FBS; Invitrogen, $\mathrm{CA}$, USA) at $37^{\circ} \mathrm{C}$ in a humidified atmosphere of $95 \%$ air and $5 \% \mathrm{CO}_{2}$. Cells were passaged according to the number of cells required for different experiments. The second day after passage, the cells were incubated with 10, 100, 250 and $500 \mu \mathrm{g} / \mathrm{ml}$ ox-LDL (Yuanye Biotechnology, Shanghai, China) and/or with $500 \mu \mathrm{M}$ salidroside (purity, $>98 \%$; Yuanye Biotechnology; $50 \mu \mathrm{M}$ pravastatin was used as a positive control) for $48 \mathrm{~h}$ to establish lipid oxidation and AS therapy models. Then, the cells were incubated with $20 \mu \mathrm{l}$ of CCK-8 (Beyotime, Shanghai, China) for $2 \mathrm{~h}$, according to the manufacturer's instructions. Cell proliferation was analyzed using a microplatereader, and the absorbance was measured at $450 \mathrm{~nm}$ (Miltenyi Biotec $\mathrm{GmbH}$, Bergisch Gladbach, Germany).

Oil Red $O$ and senescence-associated $\beta$-galactosidase (SA- $\beta$-gal) staining. Oil Red O staining was carried out to assess cellular lipid accumulation. Briefly, cells were fixed with $4 \%$ paraformaldehyde solution for $30 \mathrm{~min}$ and then stained with $0.05 \%$ Oil Red O solution in $60 \%$ isopropanol for $30 \mathrm{~min}$ at room temperature. The cells were subsequently decolorized with $75 \%$ alcohol $/ 60 \%$ isopropanol for $2 \mathrm{~min}$ before being photographed and analyzed using Image-Pro Plus 6.0 software (Media Cybernetics Inc., Rockville, MD, USA).

Senescent EA.hy926 cells express a $\beta$-gal, which was detected using a Beta Galactosidase ( $\beta$-gal) Activity Assay kit (BioVison, Milpitas, CA, USA), according to the manufacturer's instructions, based on the formation of a local blue precipitate upon enzyme-mediated cleavage. Briefly, the cells were fixed in $4 \%$ formaldehyde for $10 \mathrm{~min}$ at room temperature and washed in phosphate buffered saline (PBS). Freshly prepared aged $\mathrm{x}$-gal solution was used to stain senescent cells for approximately $18-24 \mathrm{~h}$ at $37^{\circ} \mathrm{C}$. Then, the cells were observed via lightmicroscopy, and the percentages of $\beta$-gal-positive cells were calculated.

RNA isolation and real-time PCR assay. Total RNA from EA.hy926 cells was prepared using TRIzol reagent (Invitrogen, Carlsbad, CA, USA), according to the manufacturer's instructions. Reverse transcription was performed using a PrimeScript $^{\mathrm{TM}}$ II 1st Strand cDNA Synthesis kit (Takara, Shiga Prefecture, Japan), according to the manufacturer's instructions, and cDNA was used as a template for PCR amplification of the target mRNA fragments. A SYBR ${ }^{\circledR}$ Premix Ex Taq ${ }^{\mathrm{TM}}$ (TliRNaseH Plus; Takara, Shiga Prefecture, Japan) system was used for all real-time PCR reactions. The comparative threshold cycle $\left(\Delta C_{\mathrm{q}}\right)$ method was used to quantify target mRNA levels at different time points. Target mRNA levels were normalized to an endogenous reference $18 \mathrm{~S}$ rRNA and a control. The following primer pairs were used: p66 sense, 5'-GGTTCATGT TAACCAGGCCA-3' and anti-sense, 5'-TGAGACTGCCAG ACACCAAG-3'; p53 sense, 5'-GCTTTCCACGACGGTGAC-3' and anti-sense, 5'-GCTCGACGCTAGGATCTGAC-3'); p21 sense, 5'-AGTCAGTTCCTTGTGGAGCC-3' and anti-sense, 5'-CATGGGTTCTGACGGACAT-3'; p16 sense, 5'-CCCAAC GCACCGAATAGTTA-3' and anti-sense, 5'-GGTCGGGTG AGAGTGGC-3'); and 18S rRNA sense, 5'-TAGTAGCGACGG GCGGTGTG-3' and anti-sense, 5'-CAGCCACCCGAGTTG AGCA-3').

Western blot analysis. Western blot analysis was performed as described previously (20). Primary polyclonal anti-p66, anti-p53, anti-p27, anti-p21, anti-p16, anti-CDK4, anti-cyclin D1, anti-p-Akt, anti-Akt, anti-p-Erk, anti-Erk, anti-p-Rb, anti-Rb and anti- $\beta$-actin antibodies were all purchased from Abcam (Abcam, Massachusetts, USA). After the cells were incubated with a secondary horseradish peroxidase (HRP)-conjugated goat anti-rabbit or goat anti-mouse immunoglobulin (Ig)G antibody (1:10,000; SABC) for $1 \mathrm{~h}$ at $37^{\circ} \mathrm{C}$, signals were detected using an enhanced chemiluminescence (ECL) kit (Pierce, Rockford, IL, USA) and quantified via densitometric analysis using Image-Pro Plus 6.0 software (Media Cybernetics).

Cell cycle analysis. Changes in EA.hy926 cell cycle progression induced by ox-LDL treatment were investigated by flow cytometry. Briefly, cells $\left(1 \times 10^{5}\right)$ were harvested and fixed with 

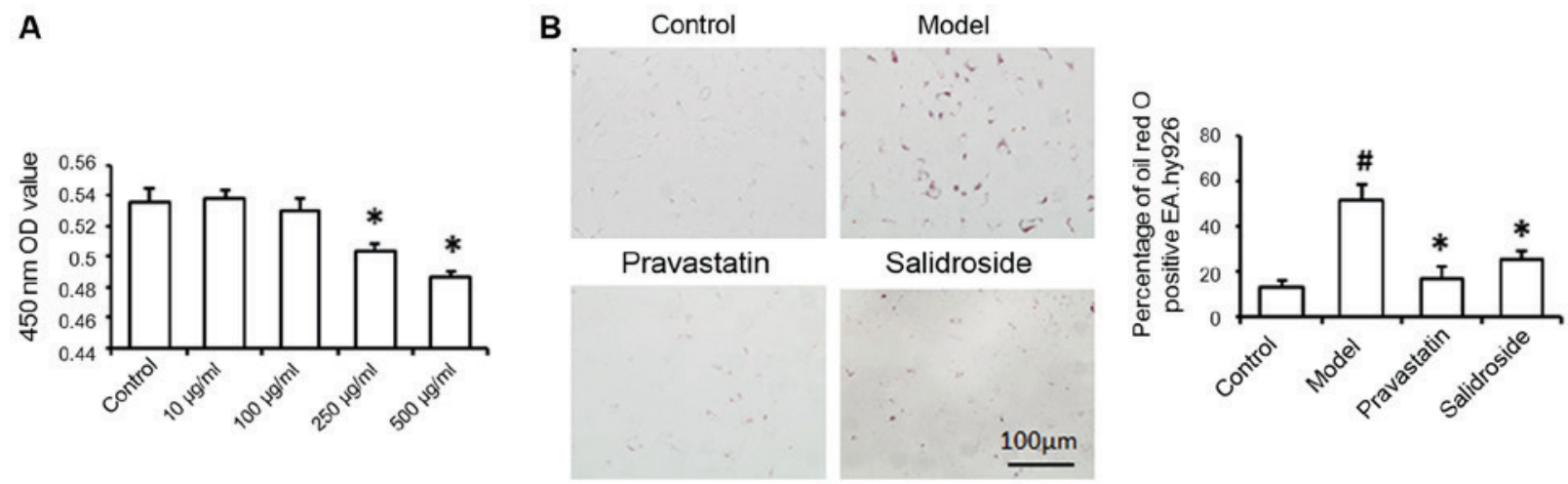

Figure 1. Effects of salidroside on ox-LDL-induced EA.hy926 cell damage. (A) Cells were treated with 10, 100, 250 and $500 \mu \mathrm{g} / \mathrm{ml}$ ox-LDL and CCK-8 assay detected the cell damage. (B) Cells were treated with $100 \mu \mathrm{g} / \mathrm{ml}$ ox-LDL alone (model) or ox-LDL, $500 \mu \mathrm{M}$ salidroside (salidroside) for $48 \mathrm{~h}$, normal cells without any treatment (control) and pravastatin (positive control). Oil Red O staining showing lipid deposition in the cell cytoplasm, and according to the staining results, we calculated the percentage of Oil Red O positive cells. Pravastatin was used as a positive control. Data represent the mean \pm standard deviation from 3 independent experiments. ${ }^{~} \mathrm{P}<0.05$ vs. control and ${ }^{*} \mathrm{P}<0.05$ vs. model. Scale bar, $100 \mu \mathrm{m}$. ox-LDL, oxidized low-density lipoprotein.

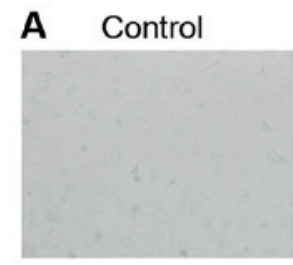

Pravastatin

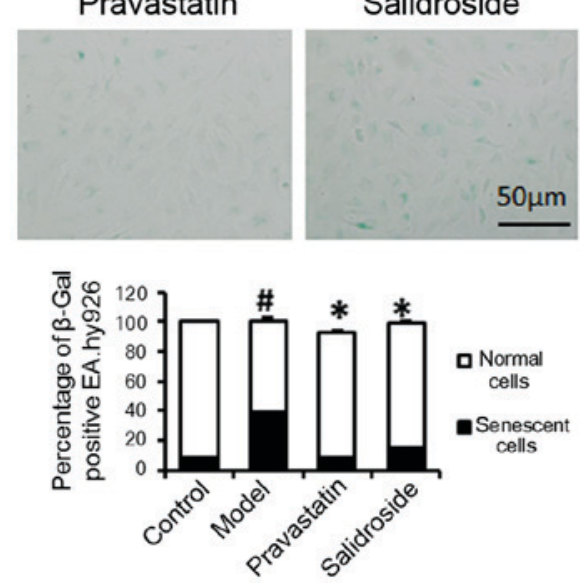

B
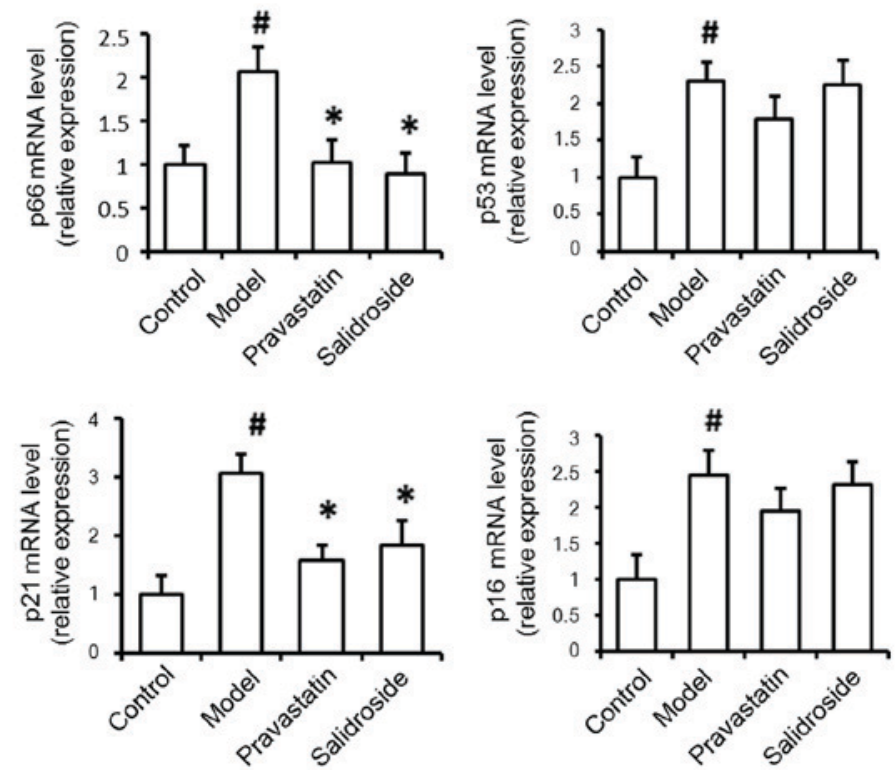

Figure 2. Salidroside treatment represses cell aging and senescence-related molecule mRNA expression. (A) EA.hy926 cells treated with $100 \mu \mathrm{g} / \mathrm{ml} \mathrm{ox}-\mathrm{LDL}$ (model) were subjected to SA- $\beta$-gal analysis. (B) Quantitative polymerase chain reaction was used to determine the relative levels of p66, p53, p21 and p16 mRNA expression in normal cells without any treatment (control), cells treated with $100 \mu \mathrm{g} / \mathrm{ml}$ ox-LDL alone (model), cells were treated with ox-LDL and $500 \mu \mathrm{M}$ salidroside (salidroside group) and pravastatin (positive control). Data represent the mean \pm standard deviation from 3 independent experiments. ${ }^{\#} \mathrm{P}<0.05$ vs. control and ${ }^{*} \mathrm{P}<0.05$ vs. model. Scale bar, $50 \mu \mathrm{m}$. ox-LDL, oxidized low-density lipoprotein; SA- $\beta$-gal, senescence-associated $\beta$-galactosidase; p53, cellular tumor antigen 53; p21, cyclin-dependent kinase inhibitor 1; p16, cyclin-dependent kinase inhibitor $2 \mathrm{~A}$.

cold $75 \%$ ethanol for $24 \mathrm{~h}$ at $-20^{\circ} \mathrm{C}$. Then, the cells were rinsed with PBS and incubated with $150 \mu 1$ of RNase A $(100 \mu \mathrm{g} / \mu \mathrm{l}$; Sigma-Aldrich, St. Louis, MO, USA) and 0.05\% Triton X-100 for $30 \mathrm{~min}$ at $37^{\circ} \mathrm{C}$ before being resuspended in $2 \mathrm{ml}$ of PBS and $100 \mu \mathrm{l}$ of propidium iodide (PI; $2.5 \mathrm{mg} / \mathrm{ml}$ ) and incubated for $30 \mathrm{~min}$ at $4^{\circ} \mathrm{C}$. Cell cycle distribution $\left(2 \times 10^{4}\right.$ cells $)$ was determined using a CytoFLEX flow cytometer (Beckman Coulter, USA), and analysis was performed using MultiCycle software (Beckman Coulter, Brea, CA, USA).

Statistical analysis. Statistical analysis was performed using SPSS 18.0 software (SPSS Inc., Chicago, USA). The results are expressed as the mean $\pm \mathrm{SD}$, as indicated in the figure. legends.
One-way ANOVA and Tukey HSD post hoc t-tests were used to determine the level of statistical significance. $\mathrm{P}<0.05$ was considered to indicate a statistically significant difference.

\section{Results}

Salidroside attenuates lipid accumulation and senescence in endothelial cells. In order to prepare AS cell model, ox-LDL at different concentration was incubated with EA.hy926 cells, finally, $100 \mu \mathrm{g} / \mathrm{ml}$ ox-LDL was used to induce lipid damage in EA.hy926 cells (Fig. 1A). At the same time, EA.hy926 cells were treated with salidroside for 2 days. To ascertain the effects of salidroside on lipid deposition and cell senescence, 
A
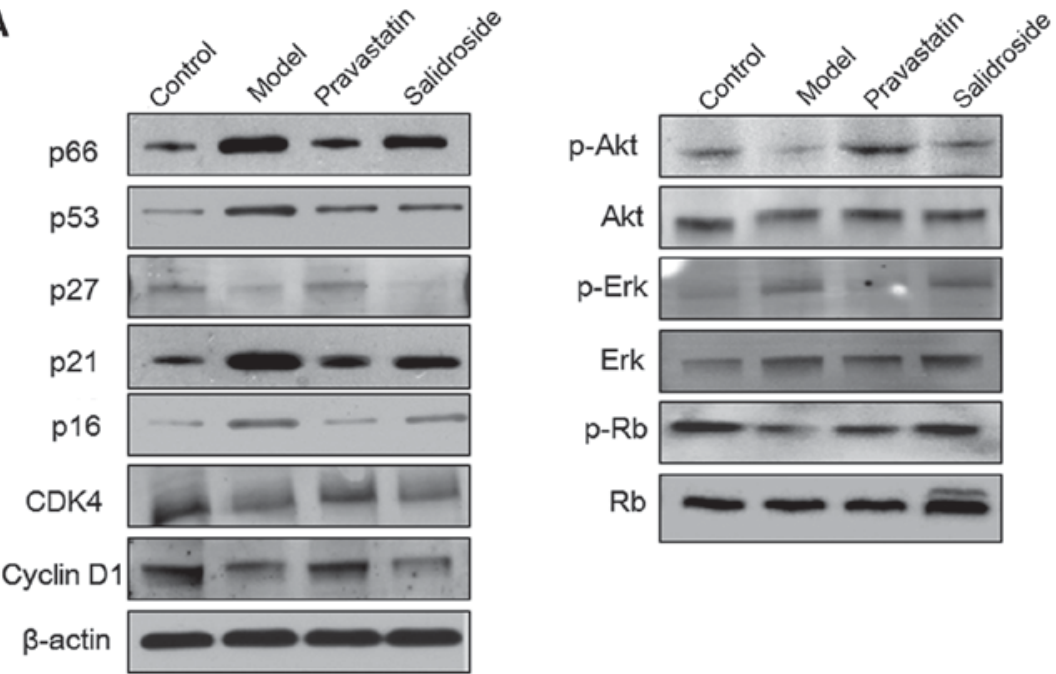

B
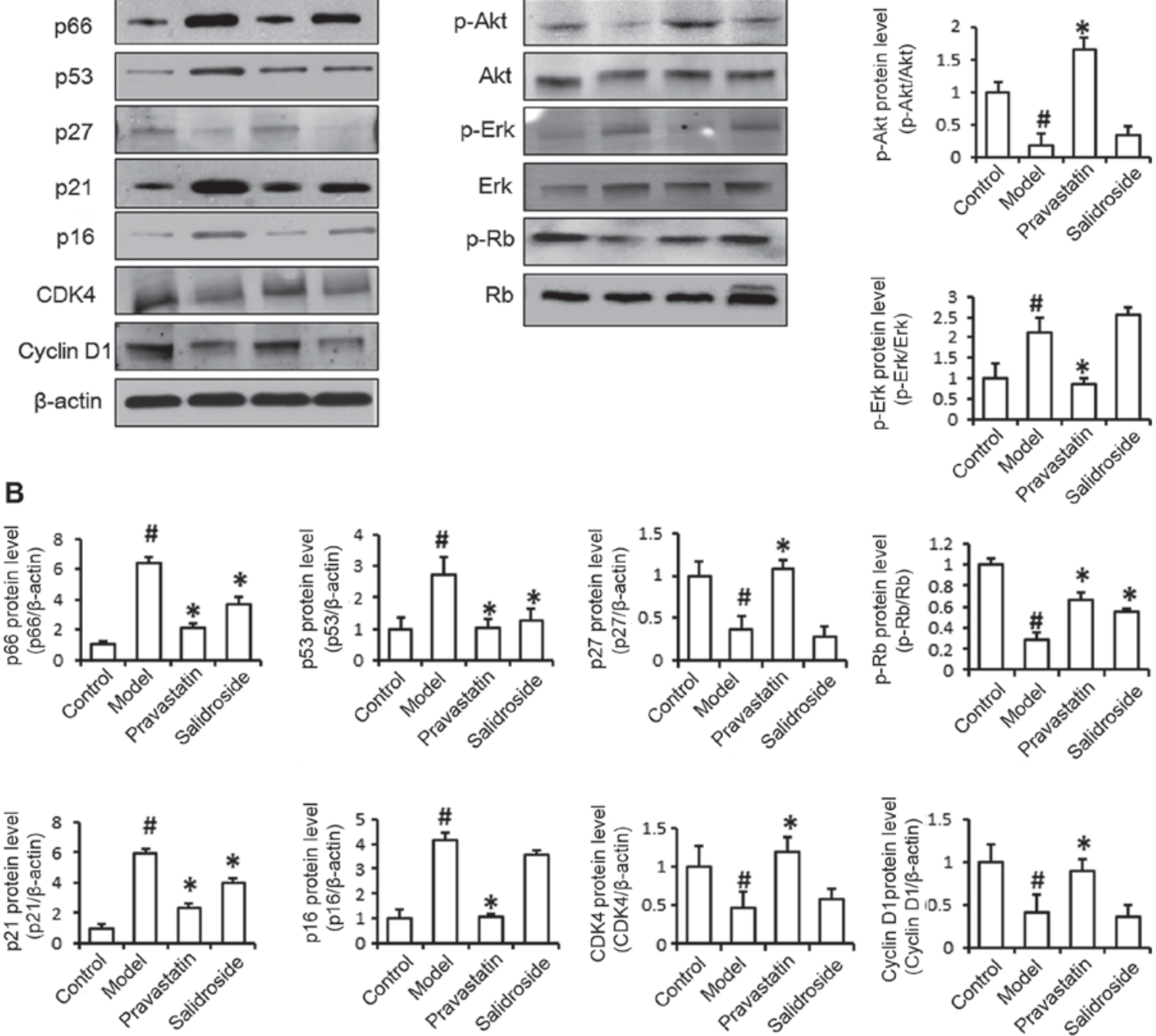

Figure 3. Salidroside treatment represses senescence-related protein expression. (A) The levels of p66, p53, p27, p21, p16, CDK-4, Cyclin D1, p-Akt, Akt, p-Erk, Erk, p-Rb and Rb protein expression were detected by western blotting. It is showed that protein levels of p66, p53 and p21 were decreased in ox-LDL (model) and salidroside-treated cells compared with ox-LDL-treated cells, protein levels of p27, p16, CDK4, Cyclin-D1, p-Akt and p-Erk have no differences between two groups. (B) The histogram of the analysis of the protein bands. Pravastatin was used as a positive control. Data represent the mean \pm standard deviation from 3 independent experiments. ${ }^{~} \mathrm{P}<0.05$ vs. control and ${ }^{*} \mathrm{P}<0.05$ vs. model. Ox-LDL, oxidized low-density lipoprotein; p53, cellular tumor antigen 53; p21, cyclin-dependent kinase inhibitor 1; p16, cyclin-dependent kinase inhibitor 2A; CDK-4, cyclin-dependent kinase-4; Akt, protein kinase B; p, phosphorylated; Erk, extracellular signal-related kinase; Rb, retinoblastoma.

Oil Red O staining and SA- $\beta$-gal staining were performed, respectively. As shown in Fig. 1B, the relative percentage of Oil Red O-positive cells was significantly lower in the salidroside group than in the ox-LDL group, indicating that intracellular lipid accumulation was successfully induced in EA.hy926 cells by ox-LDL and that salidroside treatment prevents ox-LDL-induced lipid accumulation in the cytoplasm. This finding suggests that salidroside administration attenuates lipid accumulation in endothelial cells. Histological analysis of SA- $\beta$-gal-stained cells revealed significant reductions in the numbers of positively stained cells in the salidroside-treated group compared with the ox-LDL model group (Fig. 2A). Therefore, we further examined the effect of salidroside on p66, p53, p21 and p16 mRNA expression, results indicated that the levels of p66 and p 21 mRNA expression were significantly decreased in ox-LDL plus salidroside-treated cells compared with ox-LDL-treated cells (Fig. 2B). Therefore, we hypothesized that ox-LDL-induced damage may lead to cell aging in cultured EA.hy926 cells, and salidroside improved cell senescence in ox-LDL induced cell AS model.

Salidroside decreases p66, p53 and p21 expression in ox-LDL-treated endothelial cells. We examined the effect of salidroside on senescence-related protein expression. As shown in Fig. 3, the levels of p66, p53 and p21 protein expression were significantly decreased in ox-LDL and salidroside-treated cells compared with ox-LDL-treated cells, the levels of p27, p16, CDK4, Cyclin-D1, p-Akt and p-Erk have no differences between two groups. Western blot analysis indicated that the increases in p66, p53 and p21 expression induced by ox-LDL were all significantly reversed by salidroside. The above results demonstrate that ox-LDL treatment induces EA.hy926 cell 

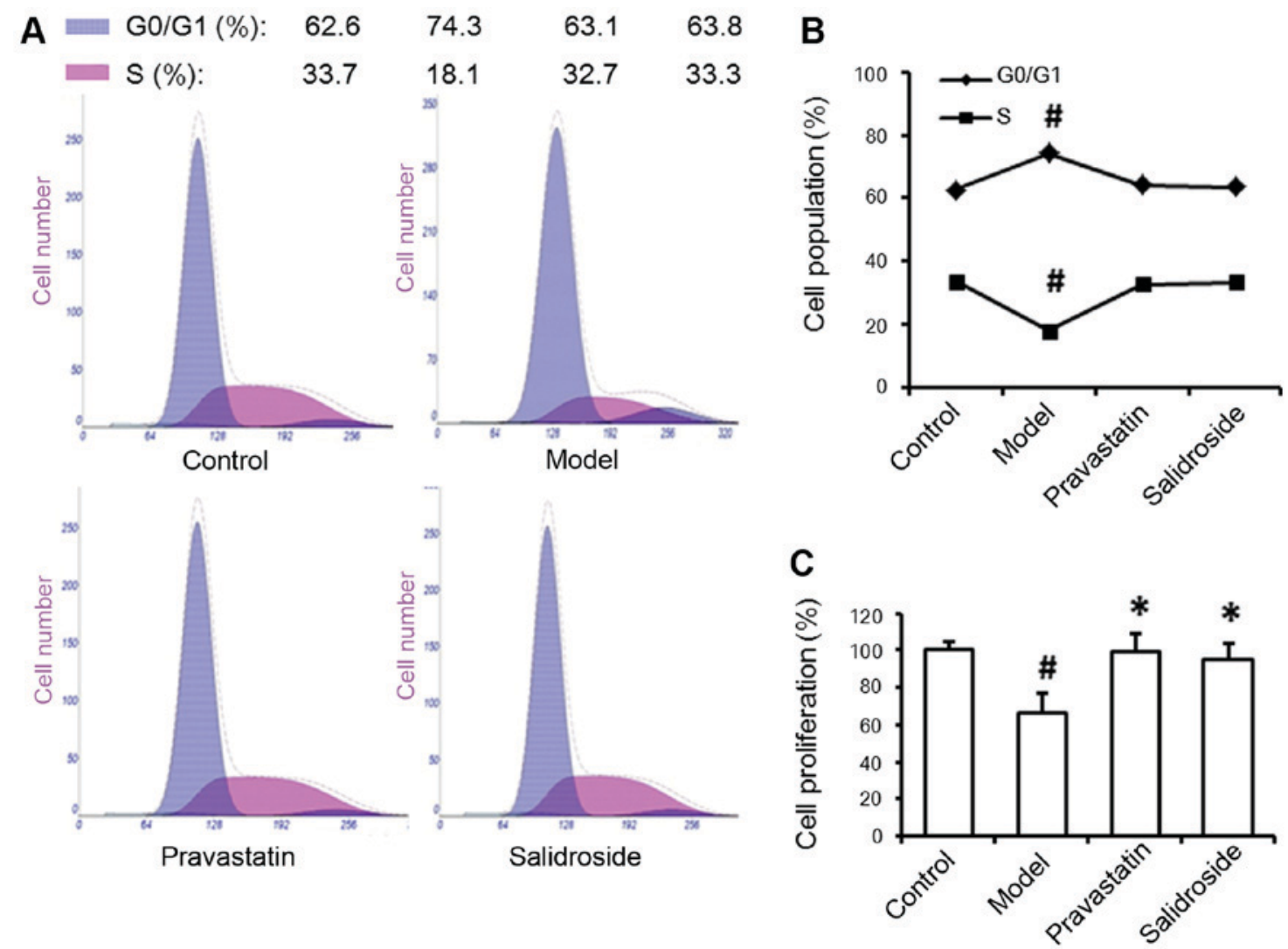

Figure 4. Salidroside influences the percent of cells in G1 and S by regulating Rb phosphorylation. (A) Fluorescence-activated cell sorting analysis of EA.hy926 cell cycle progression. (B) Quantitative analysis of cell cycle activity in cells subjected to ox-LDL treatment alone (model) or ox-LDL and salidroside treatment for 48 h. (C) Analysis of EA.hy926 cell proliferation under different culture conditions. Pravastatin was used as a positive control. Data represent the mean \pm standard deviation of 3 independent experiments. ${ }^{*} \mathrm{P}<0.05$ vs. control and ${ }^{*} \mathrm{P}<0.05$ vs. model. ox-LDL, oxidized low-density lipoprotein; Rb, retinoblastoma.

senescence and that salidroside prevents cell senescence by decreasing the expression of aging-related proteins. In order to further detect the regulation mechanisms of senescence-related protein in ox-LDL induced EA.hy926 damage, and according to previous study that the senescence-related proteins $\mathrm{p} 21$ participate in cell cycle regulation, therefore, we examined the effects of salidroside on cell cycle protein $\mathrm{Rb}$ phosphorylation to determine whether $\mathrm{Rb}$ protein is the target of salidroside. As shown in Fig. 3, phosphorylated Rb protein levels were significantly increased by salidroside treatment compared with ox-LDL treatment.

Salidroside prevents cell cycle arrest via Rb phosphorylation in senescent endothelial cells. According to the result that salidroside could phosphorylate Rb protein, we examined the effects of salidroside on cell cycle progression in ox-LDL-treated EA.hy926 cells to more fully elucidate the mechanisms by which inhibiting aging-related protein expression prevents endothelial cell senescence. In the setting of ox-LDL treatment, the percentage of EA.hy926 cells in G0/G1 phase was significantly enhanced, whereas the percentage of cells in $\mathrm{S}$ phase was markedly reduced. However, the cell cycle profile of the salidroside-treated group was characterized by an increase in the percentage of cells in $\mathrm{S}$ phase and a reduced fraction of cells in G0/G1 phase (Fig. 4A and B). Our cell proliferation assay results confirmed that salidroside also promoted EA.hy926 cell proliferation
(Fig. 4C). These results suggested that salidroside-induced senescence-related protein downregulation was involved in EA.hy926 cell cycle progression. Overall, these results indicated that salidroside regulated the cell cycle to slow cell senescence. Taken together, our findings indicate that salidroside-mediated p66, p53 and p21 regulation and $\mathrm{Rb}$ phosphorylation influence ox-LDL-induced endothelial cell senescence.

\section{Discussion}

Endothelial senescence is characterized by reduced endothelial function accompanied by vascular functional impairment and AS plaque formation. Approaches designed to retard the aging process are expected to have therapeutic value with respect to AS prevention and treatment. Salidroside is a major active ingredient from the medicinal plant Rhodiola rosea $\mathrm{L}$. and is commonly used as an antioxidant (21), anti-inflammatory (22) and an anti-lipid agent (23). Although salidroside has been shown to be useful in treating various types of diseases, its effects on vascular endothelial cell senescence-related AS development are still unclear. In the present study, we demonstrated that salidroside was capable of protecting vascular endothelial cells from lipid deposition and senescence caused by exogenous ox-LDL-induced damage. Then, we explored the possible molecular mechanisms underlying the anti-aging effects of salidroside on 
senescence-related molecule expression and found that salidroside promoted cell cycle progression from $\mathrm{G} 0 / \mathrm{G} 1$ phase to $\mathrm{S}$ phase via $\mathrm{Rb}$ phosphorylation.

Endothelial cell senescence in atherosclerotic lesions is related to the expression of various aging-related genes, which contribute to decreases in vasodilation and increases in lipid infiltration. Our results demonstrate that salidroside decreases cholesterol deposition in EA.hy926 cells and facilitates migration capacity recovery and indicate that salidroside very likely prevents cell senescence. Impaired endothelial function is the hallmark of endothelial senescence, which is mediated primarily by the aging-related proteins, which are produced by aging cells, play important roles in endothelial senescence and are involved in multiple processes that cause endothelial dysfunction, including oxidative stress and energy metabolism $(24,25)$.

In the present study, salidroside treatment decreased the protein levels of p66, p53 and p21 protein expression in ox-LDL-treated EA.hy926 cells in an in vitro AS model, but the mRNA levels of p53 did not show differences between two groups, it's mean that the posttranslational modifications might decide the 553 protein level. In addition, changes in the numbers of SA- $\beta$-gal-positive EA.hy 926 cells coincided with changes in the levels of the abovementioned aging-related proteins. These results are in accordance with those of previous studies showing the inhibitory effects of salidroside on adipogenesis (26) and cell senescence (27). The molecular mechanisms underlying cellular senescence are generally very sophisticated. According to previous reports, most researchers believe that the p53-p21 and p16-pRb pathways are the main regulators of senescence (28). These senescence-related pathways can be activated by telomere shortening, DNA damage or oxidative stress (29). The signaling pathways activated by these stresses facilitate increases in p53 and $\mathrm{Rb}$ protein expression, the combined levels of which determine whether cells enter senescence (30). However, as various endogenous and exogenous stimuli cause specific types of cell damage, the senescence pathway that is activated may vary depending on the cell-type. In our study, both the p53-p21 pathway and the p16-pRb pathway were activated in ox-LDL-treated EA.hy926 cells. We subsequently assessed cell cycle progression via PI staining and confirmed that ox-LDL-treated cells arrested in G0/G1 phase. These results indicate that Rb phosphorylation inhibits cell senescence induced by the aging-related p53-p21 and $\mathrm{p} 16-\mathrm{pRb}$ pathways. The mechanisms underlying $\mathrm{pRb}$ pathway-mediated cell growth are complex (31). In senescent cells, $\mathrm{pRb}$ dephosphorylation blocks mitogenic signaling and leaves the cells in G1 phase (32); however, the results of studies regarding $\mathrm{Rb}$ function are controversial. Additional studies regarding the molecular mechanisms underlying cell senescence are needed.

Previous studies have shown that salidroside has anti-fatigue, anti-aging,immune regulation, free radical scavenging and other pharmacological effects. Inour study, we found that salidroside ameliorates ox-LDL-induced lipid deposition, growth arrest and cell senescence in EA.hy926 cells. Its possible mechanism is that salidroside inhibits the protein expression of p53, p21 and $\mathrm{p} 16$, promotes phosphorylation of $\mathrm{Rb}$ protein, promotes cell entry into $\mathrm{S}$ phase, prevent pre-aging of cells. Non-aging cells can prevent excessive intracellular lipid deposition by accelerating cholesterol transport. Therefore, we concluded that salidroside prevented the development of AS by inhibiting cell senescence.

\section{Acknowledgements}

We would like to thank Wengong Wang (Peking University Health Science Center) for his helpful comments on the manuscript. The present study was supported by grants from Natural Science Foundation of China (program no. 81373706, 81503626) and from the Shanghai Health Bureau Youth Fund (program no. 201540254).

\section{References}

1. Hashimoto $\mathrm{J}$ and Ito $\mathrm{S}$ : Aortic stiffness determines diastolic blood flow reversal in the descending thoracic aorta: Potential implication for retrograde embolic stroke in hypertension. Hypertension 62: 542-549, 2013.

2. Li H, Horke S and Förstermann U: Vascular oxidative stress, nitric oxide and atherosclerosis. Atherosclerosis 237: 208-219, 2014

3. Tousoulis D, Oikonomou E, Economou EK, Crea F and Kaski JC: Inflammatory cytokines in atherosclerosis: Current therapeutic approaches. Eur Heart J 37: 1723-1732, 2016.

4. Shah P,Bajaj S, Virk H, Bikkina M and Shamoon F: Rapid progression of coronary atherosclerosis: A review. Thrombosis 2015: 634983, 2015.

5. Camici GG, Savarese G, Akhmedov A and Luscher TF: Molecular mechanism of endothelial and vascular aging: Implications for cardiovascular disease. Eur Heart J 36: 3392-3403, 2015.

6. Shi Y, Camici GG and Lüscher TF: Cardiovascular determinants of life span. Pflugers Arch 459: 315-324, 2010.

7. Migliaccio E, Giorgio M, Mele S, Pelicci G, Reboldi P, Pandolfi PP, Lanfrancone L and Pelicci PG: The p66shc adaptor protein controls oxidative stress response and life span in mammals. Nature 402: 309-313, 1999.

8. Shi Y, Cosentino F, Camici GG, Akhmedov A, Vanhoutte PM, Tanner FC and Lüscher TF: Oxidized low-density lipoprotein activates p66Shc via lectin-like oxidized low-density lipoprotein receptor-1, protein kinase C-beta and c-Jun N-terminal kinase kinase in human endothelial cells. Arterioscler Thromb Vasc Biol 31: 2090-2097, 2011.

9. Martin-Padura I, de Nigris F, Migliaccio E, Mansueto G, Minardi S, Rienzo M, Lerman LO, Stendardo M, Giorgio M, De Rosa G, et al: p66Shc deletion confers vascular protection in advanced atherosclerosis in hypercholesterolemic apolipoprotein E knockout mice. Endothelium 15: 276-287, 2008.

10. Zhou R, Han L, Li G and Tong T: Senescence delay and repression of p16INK4a by Lsh via recruitment of histone deacetylases in human diploid fibroblasts. Nucleic Acids Res 37: 5183-5196, 2009.

11. Skowronska-Krawczyk D, Zhao L, Zhu J, Weinreb RN, Cao G, Luo J, Flagg K, Patel S, Wen C, Krupa M, et al: P16INK4a upregulation mediated by SIX6 defines retinal ganglion cell pathogenesis in glaucoma. Mol Cell 59: 931-940, 2015.

12. Romanov VS, Pospelov VA and Pospelova TV: Cyclin-dependent kinase inhibitor p21 (Waf1): Contemporary view on its role in senescence and oncogenesis. Biochemistry (Mosc) 77: 575-584, 2012.

13. Shih CT, Chang YF, Chen YT, Ma CP, Chen HW, Yang CC, Lu JC, Tsai YS, Chen HC and Tan BC: The PPARgamma-SETD8 axis constitutes an epigenetic, p53-independent checkpoint on p21-mediated cellular senescence. Aging Cell 16: 797-813, 2017.

14. Wang M, Luo L, Yao L, Wang C, Jiang K, Liu X, Xu M, Shen N, Guo S, Sun C and Yang Y: Salidroside improves glucose homeostasis in obese mice by repressing inflammation in white adipose tissues and improving leptin sensitivity in hypothalamus. Sci Rep 6: 25399, 2016.

15. Zhang B, Wang Y, Li H, Xiong R, Zhao Z, Chu X, Li Q, Sun S and Chen $S$ : Neuroprotective effects of salidroside through PI3K/Akt pathway activation in Alzheimer's disease models. Drug Des Devel Ther 10: 1335-1343, 2016.

16. Yang ZR, Wang HF, Zuo TC, Guan LL and Dai N: Salidroside alleviates oxidative stress in the liver with non-alcoholic steatohepatitis in rats. BMC Pharmacol Toxicol 17: 16, 2016. 
17. Qi Z, Qi S, Ling L, Lv J and Feng Z: Salidroside attenuates inflammatory response via suppressing JAK2-STAT3 pathway activation and preventing STAT3 transfer into nucleus. Int Immunopharmacol 35: 265-271, 2016.

18. Xing SS, Yang XY, Zheng T, Li WJ, Wu D, Chi JY, Bian F, Bai XL, Wu GJ, Zhang YZ, et al: Salidroside improves endothelial function and alleviates atherosclerosis by activating a mitochondria-related AMPK/PI3 K/Akt/eNOS pathway. Vascul Pharmacol 72: 141-152, 2015

19. Zhao X, Jin L, Shen N, Xu B, Zhang W, Zhu H and Luo Z: Salidroside inhibits endogenous hydrogen peroxide induced cytotoxicity of endothelial cells. Biol Pharm Bull 36: 1773-1778, 2013.

20. Dou F, Huang L, Yu P, Zhu H, Wang X, Zou J, Lu P and $\mathrm{Xu} \mathrm{XM}$ : Temporospatial expression and cellular localization of oligodendrocyte myelin glycoprotein (OMgp) after traumatic spinal cord injury in adult rats. J Neurotrauma 26: 2299-2311, 2009.

21. Calcabrini C, De Bellis R, Mancini U, Cucchiarini L, Potenza L, De Sanctis R, Patrone V, Scesa C and Dachà M: Rhodiola rosea ability to enrich cellular antioxidant defences of cultured human keratinocytes. Arch Dermatol Res 302: 191-200, 2010.

22. Pooja, Bawa AS and Khanum F: Anti-inflammatory activity of Rhodiola rosea- 'a second-generation adaptogen'. Phytother Res 23: 1099-1102, 2009.

23. Wang J,Rong X,Li W, Yang Y, Yamahara J and Li Y: Rhodiolacrenulata root ameliorates derangements of glucose and lipid metabolism in a rat model of the metabolic syndrome and type 2 diabetes. J Ethnopharmacol 142: 782-788, 2012.

24. Bianchi G, Di Giulio C, Rapino C, Rapino M, Antonucci A and Cataldi A: p53 and p66 proteins compete for hypoxia-inducible factor 1 alpha stabilization in young and old rat hearts exposed to intermittent hypoxia. Gerontology 52: 17-23, 2006.

25. Noren Hooten N, Martin-Montalvo A, Dluzen DF, Zhang Y, Bernier M, Zonderman AB, Becker KG, Gorospe M, de Cabo R and Evans MK: Metformin-mediated increase in DICER1 regulates microRNA expression and cellular senescence. Aging Cell 15: 572-581, 2016.
26. Pomari E, Stefanon B and Colitti M: Effects of two different Rhodiola rosea extracts on primary human visceral adipocytes. Molecules 20: 8409-8428, 2015.

27. Mao GX, Wang Y, Qiu Q, Deng HB, Yuan LG, Li RG, Song DQ, $\mathrm{Li}$ YY, Li DD and Wang Z: Salidroside protects human fibroblast cells from premature senescence induced by $\mathrm{H}(2) \mathrm{O}(2)$ partly through modulating oxidative status. Mech Ageing Dev 131: 723-731, 2010.

28. Beauséjour CM, Krtolica A, Galimi F, Narita M, Lowe SW, Yaswen P and Campisi J: Reversal of human cellular senescence: Roles of the p53 and p16 pathways. EMBO J 22 4212-4222, 2003.

29. Martin N, Raguz S, Dharmalingam G and Gil J: Co-regulation of senescence-associated genes by oncogenic homeobox proteins and polycomb repressive complexes. Cell Cycle 12: 2194-2199, 2013.

30. Ben-Porath I and Weinberg RA: The signals and pathways activating cellular senescence. Int J Biochem Cell Biol 37: 961-976, 2005.

31. Nevins JR: The Rb/E2F pathway and cancer. Hum Mol Genet 10 699-703, 2001

32. Malumbres $M$ and Barbacid M: RAS oncogenes: The first 30 years. Nat Rev Cancer 3: 459-465, 2003.

This work is licensed under a Creative Commons Attribution-NonCommercial-NoDerivatives 4.0 International (CC BY-NC-ND 4.0) License. 